\title{
Effect of pressure on magneto-transport properties in the superconducting and normal phases of the metallic double chain compound $\operatorname{Pr}_{2} \mathrm{Ba}_{4} \mathrm{Cu}_{7} \mathrm{O}_{15-\delta}$
}

\author{
Masayoshi Kuwabara, ${ }^{1}$ Michiaki Matsukawa,, , 6 Keisuke Sugawara, ${ }^{1}$ Haruka Taniguchi, ${ }^{1}$ Akiyuki \\ Matsushita, ${ }^{2}$ Makoto Hagiwara,${ }^{3}$ Kazuhiro Sano, ${ }^{4}$ Yoshiaki Ono, ${ }^{5}$ and Takahiko Sasaki ${ }^{6}$ \\ ${ }^{1}$ Department of Materials Science and Engineering, Iwate University, Morioka 020-8551, Japan \\ ${ }^{2}$ National Institute for Materials Science, Ibaraki 305-0047 \\ ${ }^{3}$ Kyoto Institute of Technology, Kyoto 606-8585, Japan \\ ${ }^{4}$ Department of Physics Engineering, Mie University, Tsu 514-8507, Japan \\ ${ }^{5}$ Department of Physics, Niigata University, Niigata 950-2181, Japan \\ ${ }^{6}$ Institute for Materials Research, Tohoku University, Sendai 980-8577, Japan
}

(Dated: November 7, 2018)

\begin{abstract}
To examine the electronic phase diagram of superconducting $\mathrm{CuO}$ double chains, we report the effect of external pressure on the magneto-transport properties in superconducting and nonsuperconducting polycrystalline samples of $\mathrm{Pr}_{2} \mathrm{Ba}_{4} \mathrm{Cu}_{7} \mathrm{O}_{15-\delta}$ at low temperatures $(1.8-40 \mathrm{~K})$ under various magnetic fields (up to $14 \mathrm{~T}$ ). In the as-sintered non-superconducting sample, the magnetoresistance (MR) follows a power law of $H^{3 / 2}$ at low temperatures, which is in no agreement with the $H^{2}$ dependence of $\mathrm{MR}$ in the $\mathrm{PrBa}_{2} \mathrm{Cu}_{4} \mathrm{O}_{8}$ system. The negative pressure dependence of the superconducting phase is qualitatively consistent with a theoretical prediction on the basis of the Tomonaga-Luttinger Liquid theory. The 48-h-reduced superconducting sample at ambient pressure exhibits no clear increase in MR for $T>T_{c, \text { on }}=26.5 \mathrm{~K}$. In contrast, with the application of pressure to the superconducting sample, the MR effects reappear and are also well fitted by $H^{3 / 2}$. The model of slightly warped Fermi surfaces explains not only the MR effect of the non-superconducting sample, but is also related to the reasons for the pressure-induced MR phenomena of the superconducting sample.

PACS numbers: $74.25 . \mathrm{Ha}, 74.25 . \mathrm{F}-, 74.90 .+\mathrm{n}$
\end{abstract}

\section{INTRODUCTION}

Since the discovery of high- $T_{\mathrm{c}}$ copper-oxide superconductors, researches have focused on the unconventional superconductivity on the two-dimensional $\mathrm{CuO}_{2}$ planes in some cuprates. In quasi one-dimensional (1D) ladder system without $\mathrm{CuO}_{2}$ planes, it is well known that the superconductivity at $T_{\mathrm{c}}=12 \mathrm{~K}$ appears only under the application of high pressure $\stackrel{1}{-}$ In our previous study of Pr-based cuprates with metallic $\mathrm{CuO}$ double chains and insulating $\mathrm{CuO}_{2}$ planes, $\mathrm{Pr}_{2} \mathrm{Ba}_{4} \mathrm{Cu}_{7} \mathrm{O}_{15-\delta}$ is found to be a new superconductor with a higher $T_{\mathrm{c}}(15 \mathrm{~K})$ after a reduction treatment $\stackrel{2}{=}$ A nuclear quadrupole resonance (NQR) study has revealed that the newly discovered superconductivity is realized at the $\mathrm{CuO}$ doublechain block $\stackrel{3}{\underline{3}}^{-}$

Structurally, the Pr-based cuprates, $\mathrm{PrBa}_{2} \mathrm{Cu}_{3} \mathrm{O}_{7-\delta}$ (Pr123) and $\mathrm{PrBa}_{2} \mathrm{Cu}_{4} \mathrm{O}_{8}$ (Pr124), are identical to their corresponding Y-based high- $T_{\mathrm{c}}$ superconductors, $\mathrm{YBa}_{2} \mathrm{Cu}_{3} \mathrm{O}_{7-\delta}$ (Y123) and $\mathrm{YBa}_{2} \mathrm{Cu}_{4} \mathrm{O}_{8}$ (Y124). Pr123 and $\mathrm{Pr} 124$ compounds have insulating $\mathrm{CuO}_{2}$ planes and are non-superconductive $\stackrel{4,5}{ \pm}$ The suppression of superconductivity in the Pr substitutes has been explained by the hybridization of $\operatorname{Pr}-4 f$ and $\mathrm{O}-2 p$ orbitals $\underline{\underline{6}}$ The crystal structure of $\operatorname{Pr} 124$ with $\mathrm{CuO}$ double chains differs from that of $\mathrm{Pr} 123$ with $\mathrm{CuO}$ single chains. It is well known that $\mathrm{CuO}$ single chains in $\mathrm{Pr} 123$ and $\mathrm{CuO}$ double chains in Pr124 show semiconducting and metallic behaviors, respectively $\underline{\underline{7}}$ The carrier concentration of doped double chains of Pr124 is difficult to vary, because it is thermally stable up to high temperatures.

The compound $\mathrm{Pr}_{2} \mathrm{Ba}_{4} \mathrm{Cu}_{7} \mathrm{O}_{15-\delta}(\operatorname{Pr} 247)$ is an intermediate between Pr123 and Pr124. In this compound, $\mathrm{CuO}$ single-chain and double-chain blocks are alternately stacked along the $c$-axis such as $\{$-D-S-D-S- $\}$ sequence $e^{8.9}$ (see Fig 1). Here, $\mathrm{S}$ and D denote $\mathrm{CuO}$ single-chain and double-chain blocks along the $b$-axis, respectively. The physical properties of the metallic $\mathrm{CuO}$ double chains can be examined by controlling the oxygen content along the semiconducting $\mathrm{CuO}$ single chains. Anisotropic resistivity measurements of single-crystal Pr124 have revealed that metallic transport arises by the conduction along the $\mathrm{CuO}$ double chains $\underline{\underline{10}}$ In oxygen removed polycrystalline $\mathrm{Pr}_{2} \mathrm{Ba}_{4} \mathrm{Cu}_{7} \mathrm{O}_{15-\delta}$, the superconductivity appears at an onset temperature $T_{\text {c,on }}$ of $\sim 15 \mathrm{~K}^{2} \stackrel{2}{2}$ Hall coefficient measurements of superconducting $\operatorname{Pr} 247$ with $T_{\mathrm{c}, \text { on }}=15$ $\mathrm{K}$ have revealed that at intermediate temperatures below $120 \mathrm{~K}$, the main carriers change from holes to electrons, as the temperature decreases. Accordingly, this

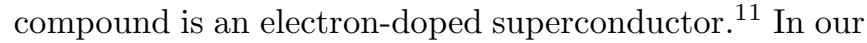
previous study, we examined the effect of magnetic fields on the superconducting phase of $\operatorname{Pr} 247 \stackrel{12}{=}$ Despite of the resistive drop associated with the superconducting transition, we found that the diamagnetic signal was strongly suppressed as expected in the $1 \mathrm{D}$ superconductivity of $\mathrm{CuO}$ double chains. We also reported the temperature dependence of the Hall coefficient in superconducting Pr247 with a higher $T_{\mathrm{c} \text {,on }} \sim 27 \mathrm{~K}: \underline{13}$ Our findings indicated that the superconducting transition temperature increased because the density of doped electron carriers 
became denser under the reduction treatment, consistent with a theoretical prediction $\underline{\underline{14}}$

In this paper, we demonstrate the magneto-transport properties of the electron-doped metallic double-chain compound $\mathrm{Pr}_{2} \mathrm{Ba}_{4} \mathrm{Cu}_{7} \mathrm{O}_{15-\delta}$, which has a higher $T_{\text {c,on }}$ $(26.5 \mathrm{~K})$, under the application of different hydrostatic pressures (up to $1.6 \mathrm{GPa}$ ). Section II outlines the experimental methods, and Sec. III presents the magneto-transport properties of superconducting and non-superconducting polycrystalline Pr247 samples under external pressures. These results are discussed in the view point of topologies of Fermi surfaces, which are varied from warped to straight form upon increasing the carrier content. The final section is devoted to a summary.

\section{EXPERIMENT}

Polycrystalline samples of $\mathrm{Pr}_{2} \mathrm{Ba}_{4} \mathrm{Cu}_{7} \mathrm{O}_{15-\delta}(\operatorname{Pr} 247)$ were synthesized by the citrate pyrolysis method. ${ }^{15}$ After several annealing processes, the resulting precursors were pressed into a pellet and calcined at $875-887^{\circ} \mathrm{C}$ for an extended period over 120-180 h under ambient oxygen pressure. The oxygen in the as-sintered sample was removed by reduction treatment in a vacuum at 500 ${ }^{\circ} \mathrm{C}$ for $48 \mathrm{~h}$, yielding a superconducting material. Typical dimensions of the pelletized rectangular sample were $9.9 \times 2.4 \times 1.7 \mathrm{~mm}^{3}$. X-ray diffraction data revealed that the as-sintered polycrystalline samples are an almost single phase with an orthorhombic structure $(\mathrm{Ammm})$, as shown in Fig.1. The lattice parameters of the as-sintered sample are $a=3.8753 \AA, b=3.9107 \AA$, and $c=50.713$ $\AA$, which are in fair agreement with those obtained by a previous study $\underline{\underline{16}}$. In a previous TEM image of superconducting Pr247, we observed a regular stacking structure of $\{-\mathrm{D}-\mathrm{S}-\mathrm{D}-\mathrm{S}-\}$ along the $c$-axis direction 17 . The oxygen deficiency in the sample with $T_{\mathrm{c}, \text { on }}=26.5 \mathrm{~K}$ prepared by the citrate method was estimated to be $\delta=0.56$ from gravimetric analysis. As a function of the oxygen deficiency, the $T_{\mathrm{c} \text {, on }}$ rises rapidly at $\delta \geq \sim 0.2$, then monotonically increases with increasing $\delta$, and finally saturates around $26-27 \mathrm{~K}$ at $\delta \geq \sim 0.6 .18$ Accordingly, the carriers in the present sample are concentrated around the optimally doped region.

The electric resistivity in zero magnetic field was measured by the $d c$ four-terminal method. The magnetotransport up to $9 \mathrm{~T}$ was measured by the ac fourprobe method using a physical property measuring system (PPMS, Quantum Design), increasing the zero-fieldcooling (ZFC) temperatures from $2 \mathrm{~K}$ to $40 \mathrm{~K}$. The high field resistivity (up to $14 \mathrm{~T}$ ) was measured in a superconducting magnet at the High Field Laboratory for Superconducting Materials, Institute for Materials Research, Tohoku University. The electric current $I$ was applied longitudinally to the sample ; consequently, the applied magnetic field $H$ was transverse to the sample (because $H \perp I$ ). Hydrostatic pressures in the electric resistivity measurement were applied using a clamp-type $\mathrm{CuBe} / \mathrm{NiCrAl}$ cell up to 1.6 GPa. Fluorinert was used as a pressure transmitting medium. Inner and outer cylinders were made of $\mathrm{NiCrAl}$ and $\mathrm{CuBe}$ alloys, respectively.

The $d c$ magnetization was performed under ZFC in a commercial superconducting quantum interference device magnetometer (Quantum Design, MPMS). The magnetization measurement under applied pressure was conducted using a standard clamp-type pressure device. A simple clamp cell consists of a $\mathrm{ZrO}_{2}$ piston and cylinder made of a CuBe alloy. The $M / H$ values of the clamp-type cell are estimated to be $\sim 10^{-8} \mathrm{emu} / \mathrm{g}$ and are considerably smaller than the values of $M / H$ for all samples.

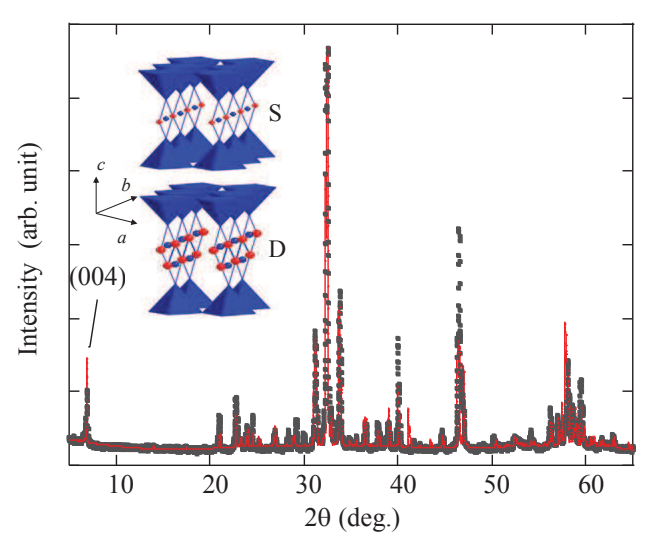

Fig. 1: (color online) X-ray diffraction patterns of as-sintered polycrystalline $\mathrm{Pr}_{2} \mathrm{Ba}_{4} \mathrm{Cu}_{7} \mathrm{O}_{15-\delta}$. The (004) peak corresponds to one of typical Miller indexes of Pr247. The calculated curve is obtained using the lattice parameters in the text. Inset shows the crystal structure of Pr247. S and D denote $\mathrm{CuO}$ single-chain and double-chain blocks along the $b$-axis, respectively.

\section{RESULTS AND DISCUSSION}

Figure 2 shows the temperature dependences of electric resistivities of the as-sintered non-superconducting and 48-h-reduced superconducting samples measured under various pressures ((a)0.GPa and $0.8 \mathrm{GPa}$, (b) $0 \mathrm{GPa}$, $0.4 \mathrm{GPa}, 0.8 \mathrm{GPa}, 1.2 \mathrm{GPa}$, and $1.6 \mathrm{GPa})$. For comparison, inset of Fig 2 plots low-temperature dependences of magnetic susceptibilities $\chi$ of the superconducting sample under various pressures (up to $1.2 \mathrm{GPa}$ ). First of all, the superconductivity of the 48-h-reduced sample was suppressed with increasing the applied pressure. When applied pressures exceed $0.8 \mathrm{GPa}$, zero-resistance state of the 48-h-reduced sample then disappeared. Second, the high-temperature metallic properties of the reduced superconducting sample were changed to the semiconducting behaviors at applied pressures above $0.8 \mathrm{GPa}$, accompanied by a rapid increase in $\rho$. The onset temperature of superconducting transition $T_{\mathrm{c}, \text { on }}$ is suppressed 

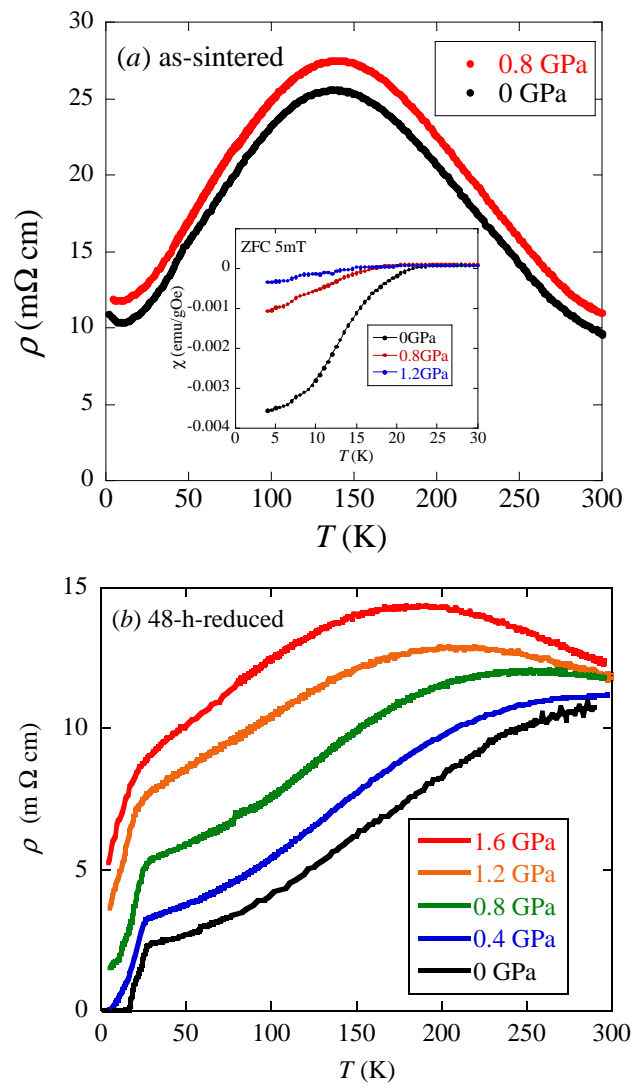

Fig. 2: (color online) (a) Temperature dependences of electric resistivities of the as-sintered non-superconducting $\mathrm{Pr}_{2} \mathrm{Ba}_{4} \mathrm{Cu}_{7} \mathrm{O}_{15-\delta}$ compound measured under $0 \mathrm{GPa}$ and 0.8 GPa. (b) Temperature dependences of electric resistivities of the 48-h-reduced superconducting $\mathrm{Pr}_{2} \mathrm{Ba}_{4} \mathrm{Cu}_{7} \mathrm{O}_{15-\delta}$ compound measured under various pressures $(0 \mathrm{GPa}, 0.4 \mathrm{GPa}, 0.8$ $\mathrm{GPa}, 1.2 \mathrm{GPa}$ and 1.6 GPa). Inset plots low-temperature dependences of magnetic susceptibilities $\chi$ of the 48-h-reduced superconducting sample measured under several pressures (up to $1.2 \mathrm{GPa})$.

from $26.5 \mathrm{~K}$ under ambient pressure through $24.1 \mathrm{~K}$ under $0.8 \mathrm{GPa}$ to $18.0 \mathrm{~K}$ under a maximum pressure of 1.6 GPa. $\left(T_{\mathrm{c}, \text { on }}\right.$ versus pressure at a zero field as displayed in inset of Fig (7) The normal-superconducting phase diagram of $\mathrm{CuO}$ double chains has been investigated on the basis of the Tomonaga-Luttinger Liquid theory $\underline{14}$ When the distance between the two single chains of a $\mathrm{CuO}$ double-chain@block shrinks along the $c$-axis, the hopping energies $t_{p p}$ and $t_{d d}$ are expected to increase. Here, $t_{p p}$ and $t_{d d}$ represent the hoping term between $2 p_{\sigma}$ orbitals at the nearest neighbor oxygen sites and that between $3 d_{x^{2}-y^{2}}$ orbitals at the nearest neighbor copper sites, respectively. The application of the external pressure on the $\mathrm{CuO}$ double chains causes an enhancement of the hopping terms, resulting in a phase transition from the superconducting to normal phase. This theoretical prediction is qualitatively consistent with the supercon-
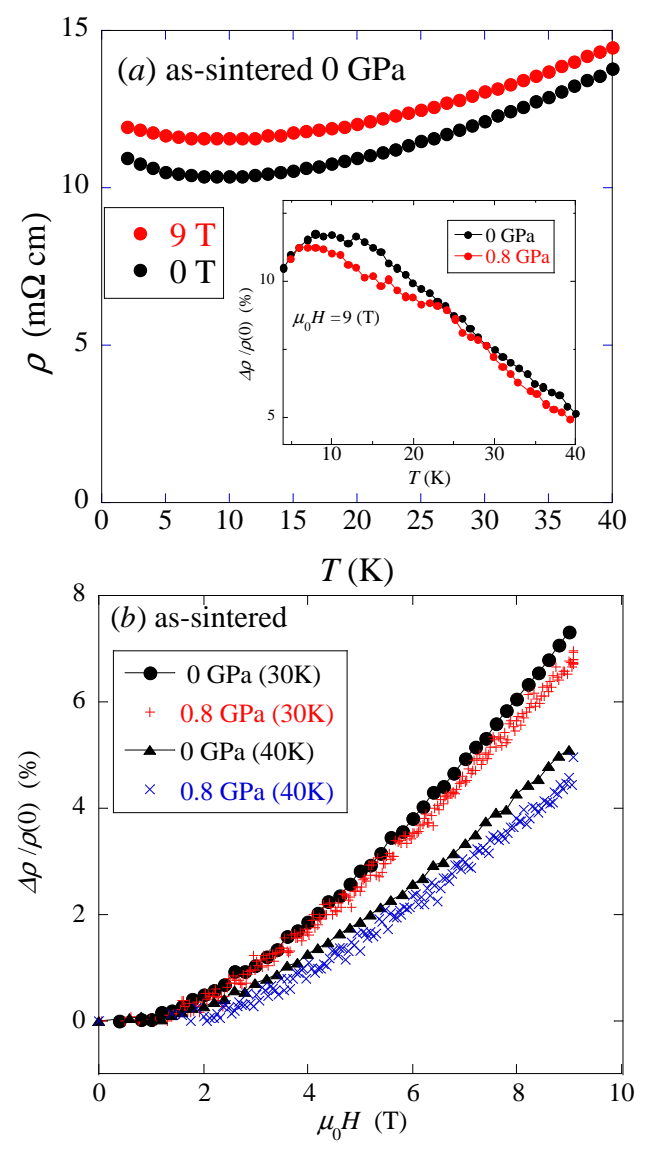

Fig. 3: (color online) (a) Low-temperature dependences of electric resistivities of the as-sintered non-superconducting $\mathrm{Pr}_{2} \mathrm{Ba}_{4} \mathrm{Cu}_{7} \mathrm{O}_{15-\delta}$ compound measured under a zero field and an applied field of $9 \mathrm{~T}$. Inset plots the magneto-resistance data of the as-sintered sample $\Delta \rho / \rho(0)$ versus $T$ (below 40 $\mathrm{K})$ under $0 \mathrm{GPa}$ and $0.8 \mathrm{GPa}$. Here, $\Delta \rho=\rho(H)-\rho(0)$. In (b), the magneto-resistance data of the as-sintered sample $\Delta \rho / \rho(0)$ are plotted as a function of $H$ (up to $9 \mathrm{~T}$ ) at $T=30$ $\mathrm{K}$ and $40 \mathrm{~K}$ under both $P=0 \mathrm{GPa}$ and $0.8 \mathrm{GPa}$.

ducting properties suppressed by the pressure effect $\underline{19}$

As shown in Fig. 2, the pressure effect on the electronic transports of the as-sintered non-superconducting sample is quite weak, in both its magnitude and temperature dependence, in comparison with that of the 48h-reduced superconducting sample. (These behaviors of the present as-sintered Pr247 are almost similar to the effect of pressure on the temperature dependence of assintered Pr247 compound prepared by high-pressure synthesis technique $\stackrel{20}{=})$

Next, let us show in Fig. 3 the temperature dependences of electric resistivities of the as-sintered nonsuperconducting $\mathrm{Pr}_{2} \mathrm{Ba}_{4} \mathrm{Cu}_{7} \mathrm{O}_{15-\delta}$ compound measured under a zero field and an applied field of $9 \mathrm{~T}$. Inset of Fig. 3 plots the magneto-resistance (MR) data of the as-sintered sample $\Delta \rho / \rho(0)$ versus $T$ (between $4 \mathrm{~K}$ and $40 \mathrm{~K}$ ) under $0 \mathrm{GPa}$ and $0.8 \mathrm{GPa}$. The value of $\Delta \rho / \rho(0)$ taken at $9 \mathrm{~T}$ reaches a maximum of about $12 \%$ near 

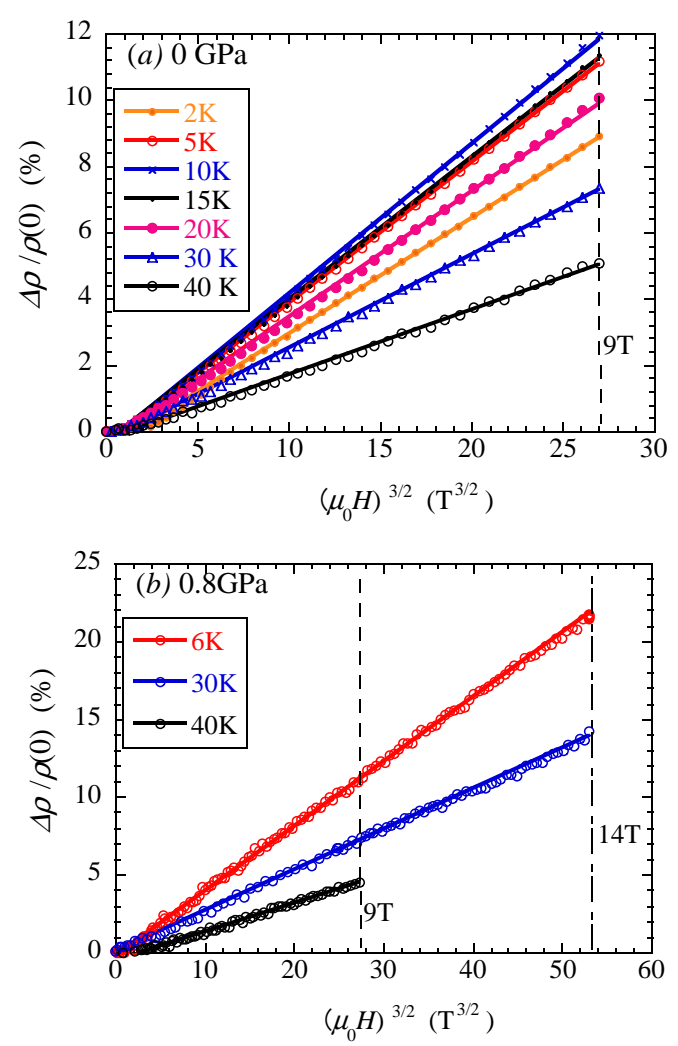

Fig. 4: (color online) Magneto-resistance effect $\Delta \rho / \rho(0)$ of the as-sintered non-superconducting $\mathrm{Pr}_{2} \mathrm{Ba}_{4} \mathrm{Cu}_{7} \mathrm{O}_{15-\delta}$ compound. In (a), the MR data (up to $9 \mathrm{~T}$ ) under ambient pressure are plotted as a function of $H^{3 / 2}$ at the selected temperatures $(T=2 \mathrm{~K}-40 \mathrm{~K})$. In (b), the MR data (up to 14 $\mathrm{T}$ ) under $0.8 \mathrm{GPa}$ are plotted as a function of $H^{3 / 2}$ at the selected temperatures of $T=5 \mathrm{~K}, 30 \mathrm{~K}$, and $40 \mathrm{~K}$.

$10 \mathrm{~K}$ under either $0 \mathrm{GPa}$ or $0.8 \mathrm{GPa}$. Moreover, the magneto-resistance curves as a function of applied field follow similar field dependences in the both cases of 0 $\mathrm{GPa}$ and $0.8 \mathrm{GPa}$. Accordingly, we infer that application of pressure on the as-sintered sample gives rise to no considerable variation in the magneto-resistance effect.

Here, we try to fit the field dependence of the MR effect $\Delta \rho / \rho(0)$ by using a power law of $H$. As shown in Fig 4 , we found out that $\Delta \rho / \rho(0)$ of the as-sintered Pr247 sample at ambient pressure is well scaled with $H^{3 / 2}$ over a wide range of temperatures $(2 \mathrm{~K}-40 \mathrm{~K})$. In a similar way, the MR data of the as-sintered sample recorded at $0.8 \mathrm{GPa}$ are well described by a power law of $\Delta \rho / \rho(0) \propto H^{3 / 2}$. Our findings are not consistent with the MR effect of polycrystalline $\operatorname{Pr} 124$ compounds with metallic double-chains. In Pr124 system, $\Delta \rho / \rho(0) \propto H^{2}$ for $H \perp I \stackrel{22}{\underline{2}}$ These discrepancies in magnetic field dependences of MR between Pr124 and Pr247 are originated from the differences of their crystal structures.
In the latter compounds, we believe that $\mathrm{CuO}$ double chains stacked along the c-axis are separated by $\mathrm{CuO}$ singe chains, resulting in a suppression of carrier hopping between individual double chains along the c-axis. Furthermore, a photoemission spectroscopy study ${ }^{23}$ has revealed that the $\mathrm{CuO}$ double chain in Pr247 has a better one dimensionality than that in $\operatorname{Pr} 124$, which is probably close to the suppression of c-axis carrier hopping.

In Pr124 system, it is well known that the interchain transport at low temperatures is destroyed by a high magnetic field applied perpendicular to the $\mathrm{CuO}$ double chains $\stackrel{24}{\underline{4}}$ The field-induced dimensional-crossover causes the weak field dependence of the c-axis magnetoresistance of $\operatorname{Pr} 124$ when the applied field exceeds the decoupling field of 11 T. Furthermore, in Y124 system, the field dependence of the c-axis magneto-resistance is changed from $H^{2}$ to $H$-linear dependence at high fields, suggesting the field-induced dimensional crossover ${ }^{25} \mathrm{An}$ anomalous field dependence of the $c$-axis $\operatorname{MR}(\Delta \rho \propto$ $H^{3 / 2}$ ) has been reported in the 1D organic conductor (TMTSF) ${ }_{2} \mathrm{PF}_{6}$ once the inter-chain transport becomes incoherent $\stackrel{26}{=}$ For Pr124 or Y124 with \{-D-D-D-D-\} sequence, the c-axis hopping of carriers between $\mathrm{CuO}$ double chains is suppressed by the application of highly magnetic field. In Pr247 system, the c-axis carrier transport is originally incoherent because of regular stacking structures of $\{-\mathrm{D}-\mathrm{S}-\mathrm{D}-\mathrm{S}-\}$ along the $c$-axis direction, as depicted in Fig 1.

Figure 5 (a) plots the low-temperature dependences of electric resistivities of the superconducting $\mathrm{Pr}_{2} \mathrm{Ba}_{4} \mathrm{Cu}_{7} \mathrm{O}_{15-\delta}$ compound with $T_{c, \text { on }}=26.5 \mathrm{~K}$ in several applied fields $(0$ T, 3 T, 6 T, 10 T, and 14 T). Even at high fields above $10 \mathrm{~T}$, the resistive drop associated with the superconducting transition is observed but the diamagnetic signal is strongly suppressed at low field below $1 \mathrm{~T}$. This observation is closely related to the $1 \mathrm{D} \mathrm{su}-$ perconductivity of $\mathrm{CuO}$ double-chains as highlighted in our previous paper $\stackrel{12}{=}$ The magneto-resistance effect (up to $14 \mathrm{~T}$ ) of the 48 -h-reduced superconducting sample in the normal sate temperature region above $T_{c, o n}=26.5$ $\mathrm{K}$ is very small in comparison to the large MR effect of the as-sintered sample. $\Delta \rho / \rho$ of the as-sintered sample recorded at $30 \mathrm{~K}$ attains $\sim 7 \%$ up to $9 \mathrm{~T}$ while for the 48-h-reduced sample the MR effect rapidly decreases below $\sim 1 \%$ at $9 \mathrm{~T}$. The suppressed MR effect in the normal phase of the 48-h-reduced superconducting sample is probably explained by the topology of Fermi surfaces of $\mathrm{CuO}$ double chains 27 , where the Fermi surfaces are changed from their warped to straight forms upon increasing carrier contents. (as mentioned below)

Figure 6 plots the low-temperature dependences of electric resistivities of the superconducting $\mathrm{Pr}_{2} \mathrm{Ba}_{4} \mathrm{Cu}_{7} \mathrm{O}_{15-\delta}$ compound measured in the several applied fields $(0 \mathrm{~T}-14 \mathrm{~T})$ under various hydrostatic pressures ((a) $P=0.4 \mathrm{GPa}$, (b) $P=1.2 \mathrm{GPa}$, and (c) $P$ $=1.6 \mathrm{GPa})$. For the superconducting sample without magnetic field, the superconducting transition temperature reaching zero-resistance state $T_{c, z e r o}$ is suppressed 

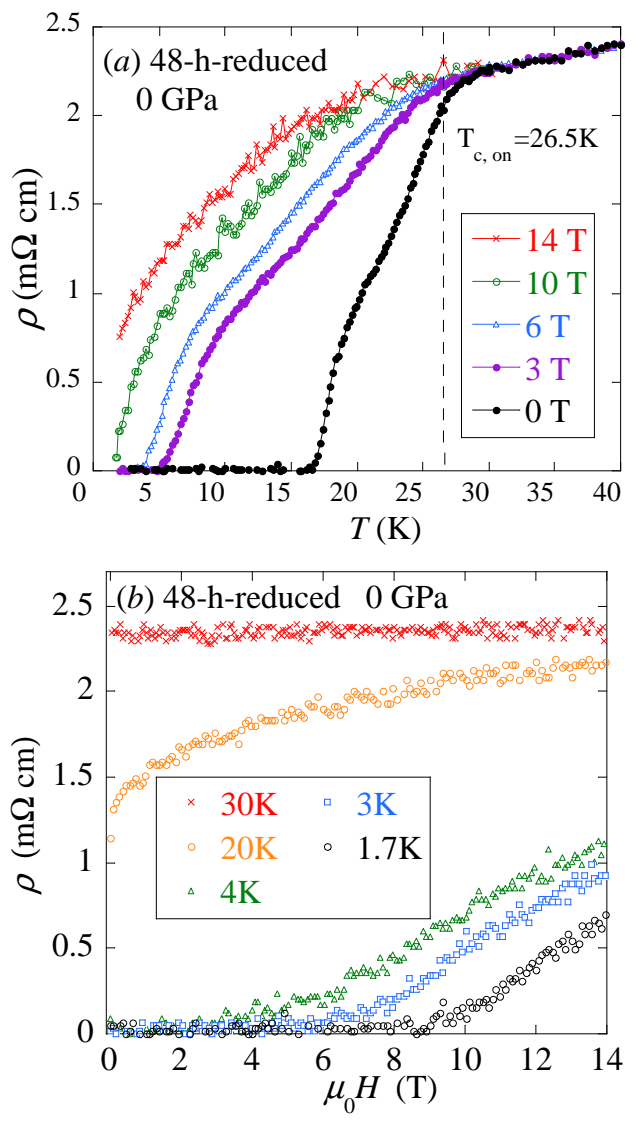

Fig. 5: (color online) (a) Low-temperature dependences of electric resistivities of the 48-h-reduced superconducting $\mathrm{Pr}_{2} \mathrm{Ba}_{4} \mathrm{Cu}_{7} \mathrm{O}_{15-\delta}$ compound measured at the several applied fields $(H=0 \mathrm{~T}-14 \mathrm{~T})$. In (b), the magneto-resistance data of the superconducting sample are plotted as a function of magnetic fields (up to $14 \mathrm{~T}$ ) at the selected temperatures $(T=$ $1.7 \mathrm{~K}-30 \mathrm{~K})$.

from $16 \mathrm{~K}$ at $0 \mathrm{GPa}$ down to $6 \mathrm{~K}$ at a low pressure of $0.4 \mathrm{GPa}$. At high fields above $10 \mathrm{~T}$, the low-temperature data under $0.4 \mathrm{GPa}$ remain the resistive drops associated with the superconducting transition, upon decreasing temperature. These tendencies still appear for the high-field resistive data (up to $10 \mathrm{~T}$ ) under both 1.2 GPa and 1.6 GPa.

For temperatures close to $T_{c, o n}$, we are concentrated on the magneto-transport properties in the normal state of the 48-h-reduced superconducting sample. As mentioned before, although the strong MR effect is observed in the as-sintered non-superconducting sample, the 48-hreduced annealing procedure for it causes a considerably suppression of the normal-state MR accompanied by the appearance of the superconductivity with $T_{c, \text { on }}=26.5$ $\mathrm{K}$. The application of pressure on the superconducting sample gives rise to a substantial decrease of $T_{c, o n}$ from $26.5 \mathrm{~K}$ at $0 \mathrm{GPa}$ down to $T_{c, o n}=18 \mathrm{~K}$ at $1.6 \mathrm{GPa}$, as shown in inset of Fig. 7. On the other hand, the finite
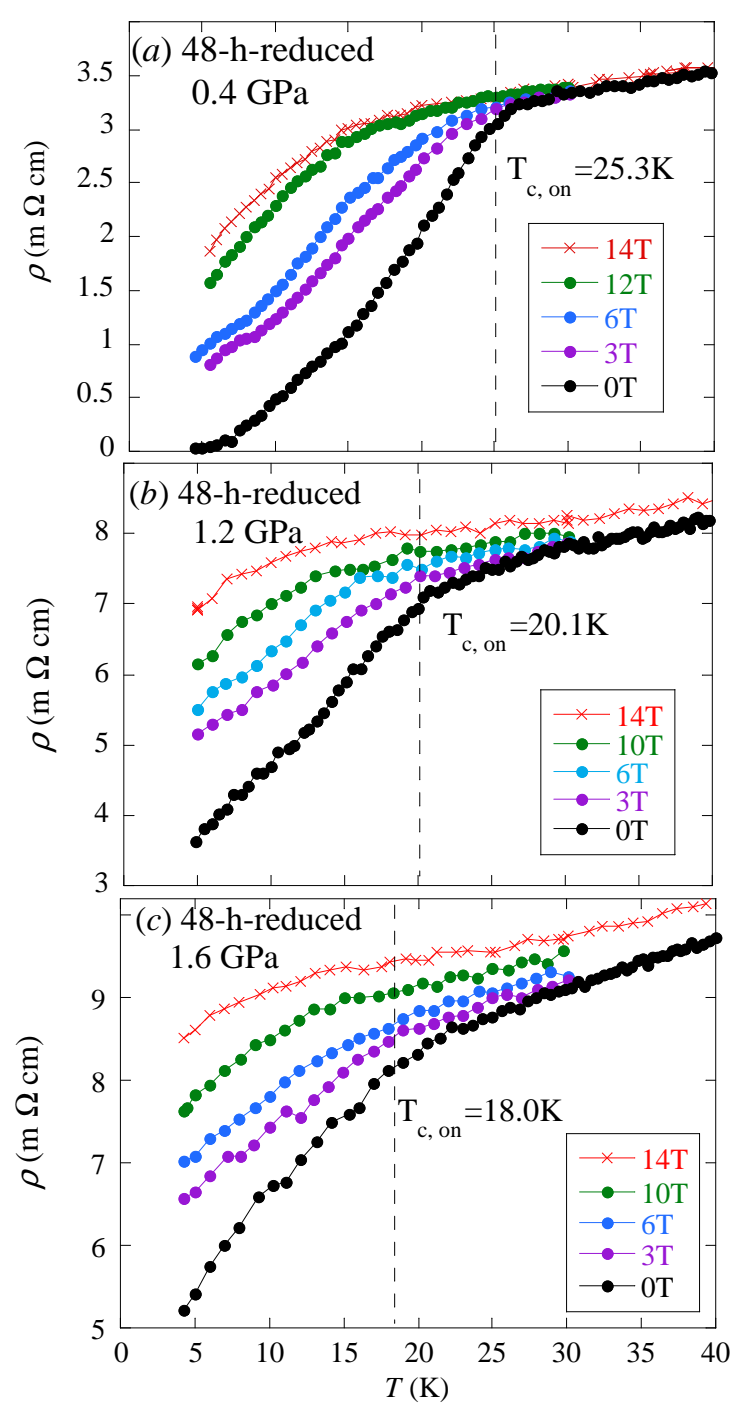

Fig. 6: (color online) Low-temperature dependences of electric resistivities of the 48-h-reduced superconducting $\mathrm{Pr}_{2} \mathrm{Ba}_{4} \mathrm{Cu}_{7} \mathrm{O}_{15-\delta}$ compound measured at several applied fields under hydrostatic pressures of (a)0.4 GPa, (b) $1.2 \mathrm{GPa}$, and $(\mathrm{c}) 1.6 \mathrm{GPa}$. For $H=0$ Tesla, $T_{\mathrm{c}, \text { on }}$ is decreased from $25.3 \mathrm{~K}$ at $0.4 \mathrm{GPa}$ through $20.1 \mathrm{~K}$ under $1.2 \mathrm{GPa}$ to $18.0 \mathrm{~K}$ under a maximum pressure of $1.6 \mathrm{GPa}$.

magneto-resistance effect reappears for $T \geq T_{c, o n}$ when the applied pressure to the superconducting sample is increased.

Figure 7 plots the magneto-resistances (up to $14 \mathrm{~T}$ ) of the 48-h-reduced superconducting sample, $\Delta \rho / \rho(0)$, measured under a maximum pressure of $1.6 \mathrm{GPa}$. For $T>T_{c, \text { on }}=18 \mathrm{~K}, \Delta \rho / \rho(0)$ curves initially start to increase in the the downward convey forms with applied fields. On the other hand, the MR data recorded at $T$ below $T_{c, o n}=18 \mathrm{~K}$ tend to increase according to the upward covey behaviors at low fields.

In a similar way to the MR of the as-sintered sample, 

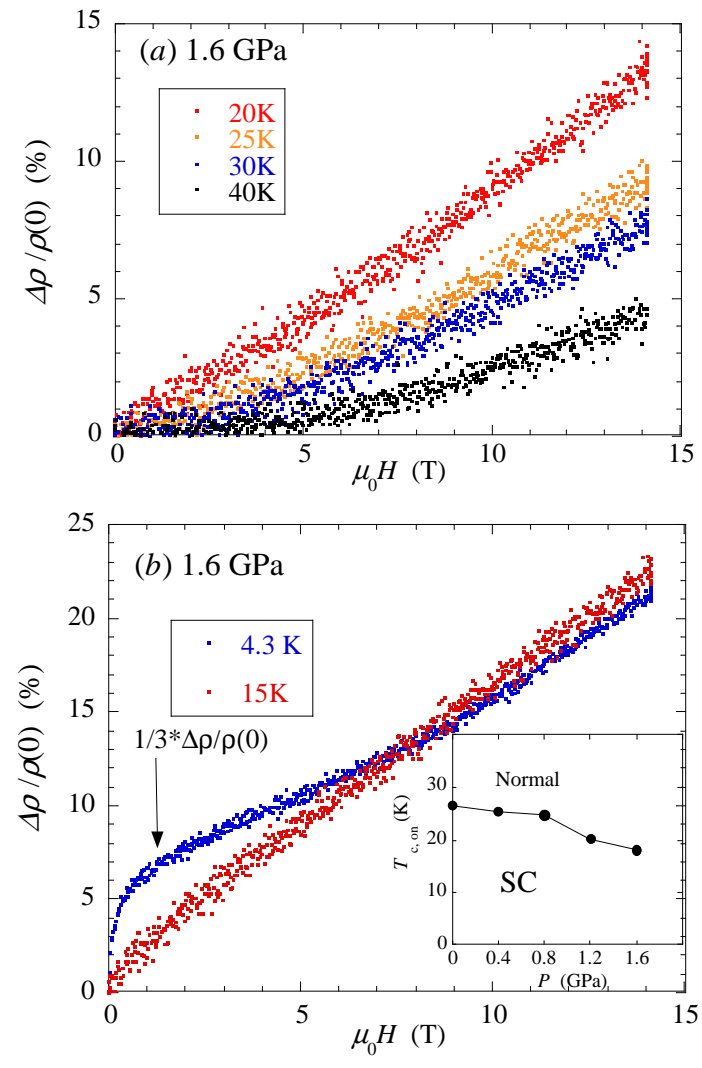

Fig. 7: (color online) Magneto-resistances (up to $14 \mathrm{~T}$ ) of the 48-h-reduced superconducting sample for temperatures close to $T_{c, \text { on }}=18 \mathrm{~K}$ under a maximum pressure of $1.6 \mathrm{GPa}$. For $T>T_{c, \text { on }}$ and $T<T_{c, \text { on }}$, the MR data are presented in (a) and (b), respectively. Inset plots $T_{c, \text { on }}$ versus pressure (up to $1.6 \mathrm{GPa})$.

the MR data of the superconducting sample recorded under 1.6 GPa are well described by a power law of $\Delta \rho / \rho(0) \propto H^{3 / 2}$ over the normal state region between $20 \mathrm{~K}$ and $40 \mathrm{~K}$, as shown in Fig 8 (a). For comparison, Fig 8 (b) shows the MR data at $T=30 \mathrm{~K}$ under $P=$ $0.4 \mathrm{GPa}, 1.2 \mathrm{GPa}$, and 1.6 GPa. The application of pressures on the superconducting sample enhances the MR at $14 \mathrm{~T}$ from $2.2 \%$ at $0.4 \mathrm{GPa}$ through $5.8 \%$ at $1.2 \mathrm{GPa}$ up to $8 \%$ at $1.6 \mathrm{GPa}$. Furthermore, the MR data under pressures are well fitted by using $\Delta \rho / \rho(0) \propto H^{3 / 2}$. (Fig. 8(b))

Now, we try to discuss the pressure-induced magnetoresistance effect of the 48-h-reduced superconducting sample. First of all, we assume that the Fermi surfaces of the double chains of the superconducting Pr247 are almost straight as indicated by the band calculation. ${ }^{28}$ In an ideal 1D conductor with straight Fermi surface without the wave number $k_{y}$, the orbitals of itinerant carries across the $\mathrm{CuO}$ double chains are not bended by the Lorentz force, resulting in no MR effect. In the case of
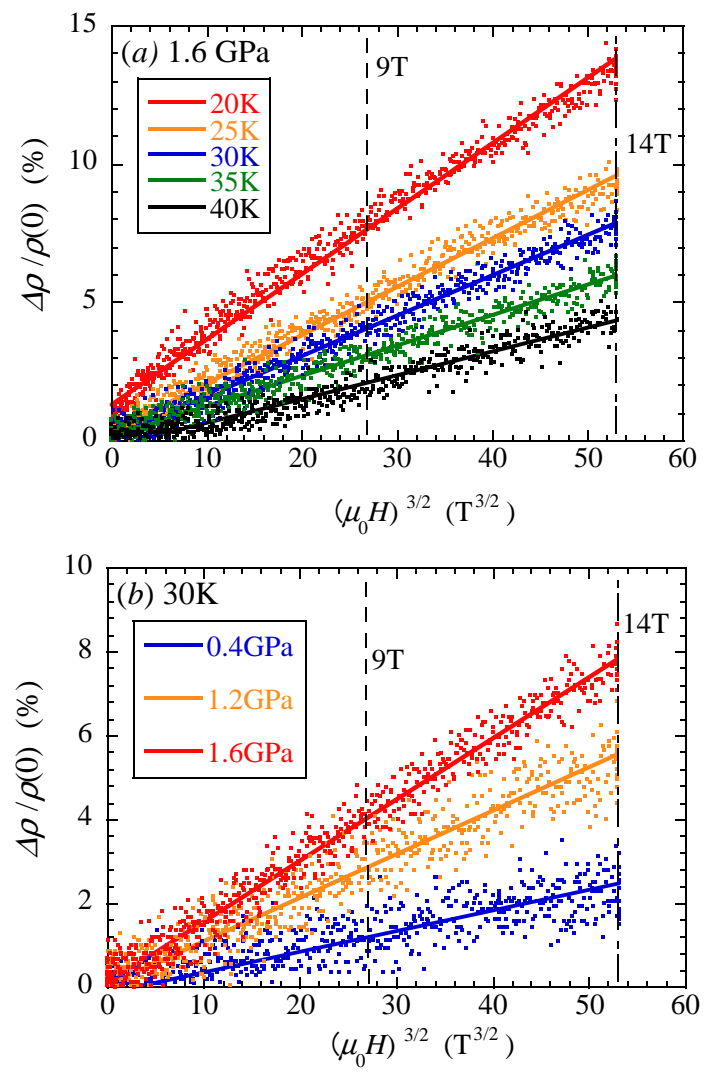

Fig. 8: (color online)The corresponding MR data (up to 14 $\mathrm{T})$ are plotted as a function of $H^{3 / 2}$. The data of (a) and (b) are collected under $P=1.6 \mathrm{GPa}$ at $T=20 \mathrm{~K}, 25 \mathrm{~K}, 30 \mathrm{~K}$, $35 \mathrm{~K}$, and $40 \mathrm{~K}$, and at $T=30 \mathrm{~K}$ under $P=0.4 \mathrm{GPa}, 1.2$ $\mathrm{GPa}$, and $1.6 \mathrm{GPa}$, respectively.

warped Fermi surface, the itinerant carriers have the finite component of wave number across the $\mathrm{CuO}$ double chain direction and the Lorentz force can bend their orbitals, giving the classical MR effect. For the superconducting $\mathrm{Pr} 247, \mathrm{CuO}$ double chains have straight Fermi surfaces at ambient pressure, which is consistent with the absence of MR effect observed. On the other hand, if the Fermi surfaces of double chains under the external pressures are changed to their warped structures with finite values of $k_{y}$. then it is naturally understood that the MR effects appear with increasing the applied pressure. In particular, the lattice shrinkage due to the hydrostatic pressure enhances not only charge transfer across two single chains of a $\mathrm{CuO}$ double chain but also increase the couplings between $\mathrm{CuO}$ double chains along the a-axis. As mentioned before, the suppressed MR effect of the superconducting sample under ambient pressure is closely related to the topology of Fermi surfaces of $\mathrm{CuO}$ double chains, which are varied from their warped to straight forms upon increasing carrier contents. Accordingly, we expect that the model of warped Fermi surfaces is not 
only applicable to the origins of the MR effect of the non-superconducting sample, but is also related to the reasons for the pressure-induced MR phenomena of the superconducting sample.

\section{SUMMARY}

We demonstrated the magneto-transport properties of superconducting and non-superconducting $\mathrm{Pr}_{2} \mathrm{Ba}_{4} \mathrm{Cu}_{7} \mathrm{O}_{15-\delta}$ compounds under the application of different hydrostatic pressures (up to $1.6 \mathrm{GPa}$ ). We found out that $\Delta \rho / \rho(0)$ of the as-sintered Pr247 sample at ambient pressure is well scaled by using $H^{3 / 2}$ over a wide range of temperatures $(2 \mathrm{~K}-40 \mathrm{~K})$. In present Pr247 system, crystal structures of $\{-\mathrm{D}-\mathrm{S}-\mathrm{D}-\mathrm{S}-\}$ along the $c$-axis direction probably make inter-chain couplings between $\mathrm{CuO}$ double chains incoherent, resulting in the $H^{3 / 2}$ dependence of MR. This result of $\operatorname{Pr} 247$ is not consistent with the $H^{2}$ dependence of MR in Pr124 system with stacking structures of $\{-D-D-D-D-\}$ along the $c$-axis. The superconducting properties suppressed by the pressure effect is qualitatively explained by the normal-superconducting phase diagram of $\mathrm{CuO}$ double chains on the basis of the Tomonaga-Luttinger Liquid theory. The 48-h-reduced annealing procedure for the as-sintered sample causes a considerably decrease in the normal-state MR accompanied by the appearance of the superconductivity with $T_{c, o n}=26.5 \mathrm{~K}$. The suppressed MR effect of the superconducting sample is probably explained by the topology of Fermi surfaces of $\mathrm{CuO}$ double chains, where the Fermi surfaces are changed from their warped to straight forms upon increasing carrier contents. In contrast, for $T>T_{c, o n}$, the application of pressure (up to $1.6 \mathrm{GPa}$ ) on the superconducting sample results in a substantial MR effect of $8 \%$ at $14 \mathrm{~T}$. In a similar way to the MR of the as-sintered sample, the MR data of the superconducting sample recorded under $1.6 \mathrm{GPa}$ are well described by a power law of $\Delta \rho / \rho(0) \propto H^{3 / 2}$ over the normal state region between $20 \mathrm{~K}$ and $40 \mathrm{~K}$. The model of slightly warped Fermi surfaces explains not only the magneto-resistance effect of the non-superconducting sample, but it also is closely related to the reasons for the pressure-induced MR phenomena of the superconducting sample.

\section{Acknowledgments}

The authors are grateful for M. Nakamura for his assistance in PPMS experiments at Center for Regional Collaboration in Research and Education, Iwate University.
* Electronic address: matsukawa@iwate-u.ac.jp

1 M. Uehara, T. Nagata, J. Akimitsu, H. Takahashi, N. Mori, and K. Kinoshita, J. Phys. Soc. Jpn., 65 (1996) 2764.

2 M. Matsukawa, Y. Yamada, M. Chiba, H. Ogasawara, T. Shibata, A. Matsushita, and Y. Takano, Physica C 411 (2004) 101.

3 S. Watanabe, Y. Yamada and S. Sasaki, Physica C 426-431 (2005) 473.

4 L. Soderholm, K. Zhang, D. G. Hinks, M. A. Beno, J. D. Jorgensen,C. U. Segre, and I. K. Schuller, Nature 328 (1987) 604.

5 S. Horii, Y. Yamada, H. Ikuta, N. Yamada, Y. Kodama, S. Katano, Y. Funahashi, S. Morii, A. Matsushita, T. Matsumoto, I. Hirabayashi, and U. Mizutani,Physica C 302 (1998) 10.

${ }^{6}$ R. Fehrenbacher and T. M. Rice, Phys. Rev. Lett. 70 (1993) 3471.

7 T. Mizokawa, C. Kim, Z. -X. Shen, A. Ino, T. Yoshida, A. Fujimori, M. Goto, H. Eisaki, S. Uchida, M. Tagami, K. Yoshida, A. I. Rykov, Y. Siohara, K. Tomimoto, S. Tajima, Yuh Yamada, S. Horii, N. Yamada, Yasuji Yamada, and I. Hirabayashi, Phys. Rev. Lett. 85 (2000) 4779.

8 P. Bordet, C. Chaillout, J. Chenavas, J. L. Hodeau, M. Marezio,J. Karpinski, and E. Kaldis, Nature 334 (1988) 596.

${ }^{9}$ Y. Yamada, S. Horii, N. Yamada, Z. Guo, Y. Kodama, K. Kawamoto,U. Mizutani, and I. Hirabayashi, Physica C 231(1994)131.

10 S. Horii, U. Mizutani, H. Ikuta, Y. Yamada, J. H. Ye, A. Matsushita, N. E. Hussey, H. Takagi, and I. Hirabayashi,
Phys. Rev. B61 (2000) 6327.

11 A. Matsushita, K. Fukuda, Y. Yamada, F. Ishikawa, S. Sekiya,M. Hedo, and T. Naka, Science and Technology of Advanced Materials 8 (2007) 477.

12 T. Chiba, M. Matsukawa, J. Tada, S. Kobayashi, M. Hagiwara, T. Miyazaki, K. Sano, Y. Ono, T. Sasaki, and J.Echigoya, J. Phys. Soc. Jpn., 82 (2013) 074706.

13 J. Tada, M. Matsukawa, T. Konno, S. Kobayashi, M. Hagiwara, T. Miyazaki, K. Sano, Y. Ono, and A. Matsushita, J. Phys. Soc. Jpn., 82 (2013) 105003.

14 K. Sano, Y. Ono, and Y. Yamada, J. Phys. Soc. Jpn., 74 (2005) 2885.

15 M. Hagiwara, T. Shima, T. Sugano, K. Koyama, and M. Matsuura, Physica C 445-448 (2006) 111.

16 Y. Yamada and A. Matsushita, Physica C 426-431 (2005) 213.

17 T. Konno, M. Matsukawa, K. Sugawara, H. Taniguchi, J. Echigoya, A. Matsushita, M. Hagiwara, K. Sano, Y. Ono, Y. Yamada, T. Sasaki, and Y. Hayasaka, Physica C 521522 (2016) 13.

18 M. Hagiwara, S. Tanaka, T. Shima, K. Gotoh, S. Kanda, T. Saito, and K. Koyama, Physica C 468 (2008) 1217.

19 F. Ishikawa, K. Fukuda, S. Sekiya, A. Kaeriyama, Y. Yamada and A. Matsushita, J. Phys. Soc. Jpn., 76 (2007) Suppl. A 92.

20 A. Matsushita, Y. Yamada, N. Yamada, S. Horii, and T. Matsumoto, Physica C 242 (1995) 381.

21 N. Wakeham, and N. E. Hussey, Phys. Rev. B85 (2012) 235117.

22 I. Terasaki, N. Seiji, S. Adachi, and H. Yamauchi, Phys. 
Rev. B54 (1996) 11993.

23 Y. Wakisaka, K. Takubo, T. Sudayama, J. Y.Son, T. Mizokawa, M. Arita, H. Namatame, M. Taniguchi, S. Sekiya, K. Fukuda, F. Ishikawa, and Y. Yamada, J. Phys. Soc. Jpn., 77 (2008) 074710.

24 N. E. Hussey, M. N. McBrien, L. Balicas, J. S. Brooks, S. Horii, and H. Ikuta, Phys. Rev. Lett. 89 (2002) 086601.

25 N. E. Hussey, M. Kibune, H. Nakagawa, N. Miura, Y. Iye, H. Takagi, and S. Adachi, and K. Tanabe, Phys. Rev. Lett.
80 (1998) 2909.

26 G. M. Danner, and P. M. Chaikin, Phys. Rev. Lett. 75 (1995) 4690.

27 T. Nakano, K. Kuroki, and S. Onari, Phys. Rev. B76 (2007) 014515.

28 T. Habaguchi, Y. Ono, H. Y. Du Gh, K. Sano, and Y. Yamada, J. Phys. Soc. Jpn., 80 (2011) 024708. 